\title{
Ewing K.D., Hendy J., Carolyn Jones, A Manifesto for Labour Law: towards a comprehensive revision of workers' rights
}

The Institute of Employment Rights, 2017

\section{Andrea Allamprese}

\section{OpenEdition}

\section{Journals}

Édition électronique

URL : https://journals.openedition.org/rdctss/2069

DOI : $10.4000 /$ rdctss.2069

ISSN : 2262-9815

Éditeur

Centre de droit comparé du travail et de la sécurité sociale

Édition imprimée

Date de publication : 1 avril 2018

Pagination : 203-204

ISSN : $2117-4350$

Référence électronique

Andrea Allamprese, «Ewing K.D., Hendy J., Carolyn Jones, A Manifesto for Labour Law: towards a comprehensive revision of workers' rights », Revue de droit comparé du travail et de la sécurité sociale [En ligne], 1 | 2018, mis en ligne le 01 novembre 2021, consulté le 12 novembre 2021. URL : http:// journals.openedition.org/rdctss/2069; DOI : https://doi.org/10.4000/rdctss.2069

\section{(c) (†) $९$}

Revue de droit comparé du travail et de la sécurité sociale est mise à disposition selon les termes de la Licence Creative Commons Attribution - Pas d'Utilisation Commerciale - Pas de Modification 4.0 International. 


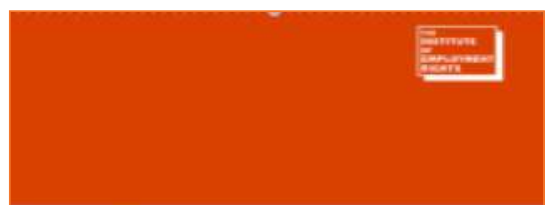

A Manifesto for Labour Law: towards a comprehensive revision of workers' rights

edited by K D Ewing, John Hendy and Carolyn Jones

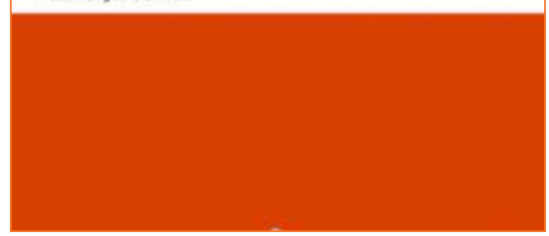

Ewing K.D., Hendy J., Carolyn Jones

A MANIFESTO FOR LABOUR LAW: TOWARDS A COMPREHENSIVE REVISION OF WORKERS' RIGHTS

The Institute of Employment Rights, 2017

\section{Andrea Allamprese}

Professeur en Droit du travail

Université de Modene et Reggio Emilia

La revendication des droits sociaux fondamentaux, comme patrimoine symbolique culturel à préserver face aux pouvoirs publics et privés, est quelque chose de profondément enraciné dans le mouvement ouvrier européen. Certaines propositions récentes de la Confederazione generale italiana del lavoro (CGIL) en Italie1, des Commissiones Obreras (CC.OO.) en Espagne2, du Trade Union Congress (TUC) au Royaume-Uni revendiquent un certain «chartisme social » à traduire en règles juridiques contraignantes dans les États nations respectifs3.

Les propositions susmentionnées sont souvent le fruit de la rencontre positive entre les syndicats et le monde des universitaires. Elles ont en commun une forte attention au thème de l'extension des droits sociaux et de leur effectivité, face au problème des «faux indépendants $"$.

L'extension du salariat aux travailleurs faussement indépendants pourrait être réalisée en complétant le critère du lien de subordination par un autre critère, celui de la dépendance économique4. C'est la proposition qui a été faite récemment en France par le Groupe de recherche pour un autre Code du travail5.

S'inscrit dans ce même giron la proposition avancée au Royaume-Uni par un groupe de quinze universitaires anglais (sous la coordination de Keith Ewing, John Hendy et Carolyn Jones). Ce groupe d'universitaires, proches du Trade Union Congress a élaboré "A Manifesto for Labour Law: towards a comprehensive revision of workers' rights", composé

1 On se réfère à la « Charte des droits universels du travail », la proposition de loi populaire présentée en 2016 par la CGIL qui comprend un projet de protections générales destinées aux travailleurs dans un proche avenir.

2 On se réfère à la « Propuesta de CCOO para un modelo más democrático de relaciones laborales y un cambio en la política económica y social», oct. 2015, page 15 et suivantes. Voir aussi «Diez propuestas para un nuevo Estatuto de los Trabajadores con 50 medidas », 21 mai 2015.

3 A. Baylos Grau, «Poteri privati e diritti fondamentali dei lavoratori », Lavoro e diritto, 1, 2017.

4 A. Supiot, "Et si l'on refondait le droit du travail... », Le Monde Diplomatique, oct. 2017.

5 E. Dockès (dir.), Proposition de Code du travail, Dalloz, 2017. Article L 11-3, Code du travail: «Le salarié est une personne physique qui exécute un travail sous le pouvoir de fait ou sous la dépendance d'autrui ». 
de 8 chapitres (the 4 pillars of collective bargaining, improving statutory protection, making rights work, securing freedom of association, enhancing the right to strike) et de plusieurs recommandations/propositions finales.

Les auteurs du Manifeste proposent de déplacer le centre de gravité de la régulation de la loi vers la négociation collective (§ 4.1): la loi établirait les standards minima égaux pour tous, alors que la négociation au niveau sectoriel aurait pour mission de régler les détails de la prestation de travail: salaires minima, horaires de travail (avec une attention particulière au problème du contrat à zéro heure), égalité (par exemple, avec l'idée imposer par voie législative aux employeurs de constituer sur le lieu de travail un "forum" de l'égalité qui permettrait d'aborder la question des discriminations avec les représentants syndicaux et la proposition également d'élection d'un "représentant pour l'égalité), santé et sécurité, etc. Une Commission du travail par branche aurait pour tâche d'élaborer des conventions collectives de branche, obligatoires pour tous ceux qui travaillent dans le secteur ( $\$ 3.13$ et suivants). Les conventions collectives d'entreprise (avec un seul employeur ou un groupe) ne pourraient contenir de dérogations que pour améliorer les conditions établies par les conventions collectives de branche (§ 3.17). Ces conventions d'entreprise ne pourraient être conclues que par des syndicats représentant au moins 10\% des travailleurs de l'entreprise (§ 3.18).

Les lois en vigueur devraient être appliquées universellement à tous les travailleurs et être effectives (§ 5.1). Par conséquent, la définition légale de «worker » (qui actuellement exclut de nombreux travailleurs précaires, allant des travailleurs temporaires aux gig workers) devrait être élargie ( $\S 5.8$ et point 11 des recommandations) et devrait être une présomption légale selon laquelle, quiconque effectue un travail en faveur d'autrui, sans exercer une activité économique pour son compte propre, devrait être considéré, sauf preuve contraire, comme un travailleur (§5.9). Les auteurs du Manifeste proposent, ensuite, de considérer que la "continuity of employment" ne soit pas interrompue chaque fois que le travailleur n'exerce aucune activité professionnelle, alors qu'une future prestation pourrait lui être demandée sur la base du contrat conclu avec l'employeur (§ 5.11).

Le domaine de la santé et de la sécurité au travail est emblématique. La révision du régime juridique exigerait de créer des obligations en matière de sécurité applicables aux différentes formes de travail non standard, via: a) la réforme du Health and Safety at Work Act de 1974 en élargissant le champ d'application de l'obligation générale de sécurité aux "personnes qui conduisent des affaires ou une entreprise" (Persons conducting a business or an undertaking - PCBU); b) l'extension de cette nouvelle obligation en matière de sécurité aux workers "engaged, or caused to be engaged, by such persons" et à ceux "whose activities in carrying out work are influenced or directed" par elles (§ 4.18).

Pour renforcer l'effectivité de la réglementation en matière de travail, les auteurs du Manifeste proposent enfin de garantir aux inspecteurs du travail et aux syndicalistes la possibilité d'agir en justice pour le compte des travailleurs (§ 5.15) et de supprimer les coûts de saisine du juge. 